\title{
Assessment Of Third Ventricular Parameters in Computed Tomography of Head: A Pilot Study in Tertiary Level Hospital
}

\author{
Umesh P Khanal, Keshav Sah, Ram B Chand \\ Department of Radiology and Imaging, Maharajgunj Medical Campus, Tribhuvan University Teaching Hospital, \\ Institute of Medicine, Maharajgunj, Kathmandu
}

\section{Corresponding author:}

Umesh P Khanal, MBBS, MD

Department of Radiology and Imaging, Maharajgunj Medical Campus, Tribhuvan University Teaching Hospital, Institute of Medicine, Maharajgunj, Kathmandu

Email: upkhanal2007@gmail.com

Submitted : Oct 8, 2020

Accepted : Nov 30, 2020

\begin{abstract}
Introduction

Measurement of ventricles of the brain is important for evaluating changes due to growth, ageing, intrinsic and extrinsic pathologies. The aim of this study was to determine the various parameters of the third ventricle and to assess their association with gender and age using computed tomography (CT) scans of head.

\section{Methods}

This retrospective, quantitative, cross sectional study was performed in the Department of Radiology and Imaging, Tribhuvan University Teaching Hospital from July to October 2019 (4 months) on images of 100 patients (50 males and 50 females) whose age group were 18-87 years, who underwent non-contrast and contrast enhanced CT scan of head.
\end{abstract}

\section{Results}

The total mean of width of third ventricle was found to be $0.33 \pm 0.09 \mathrm{~cm}, 0.35 \pm 0.10 \mathrm{~cm}$ in male and $0.31 \pm 0.06 \mathrm{~cm}$ in female. Third ventricle width (TVW) showed statistically significant correlation with male and also with Age. Mean of third ventricle sylvian fissure distance index was $0.41 \pm 0.67 \mathrm{~cm}, 0.42 \pm 0.65 \mathrm{~cm}$ in male and $0.39 \pm 0.06 \mathrm{~cm}$ in female respectively. Mean of third ventricle ratio was $0.03 \pm 0.09 \mathrm{~cm}, 0.03 \pm 0.09$ $\mathrm{cm}$ in male and $0.02 \pm 0.09$ in female respectively. The value were slightly higher in male.

\section{Conclusion}

The third ventricle width in CT in this study was $0.33 \pm 0.09 \mathrm{~cm}$. CT was very useful to determine the upper limits of normal value and its variation with age.

\section{Keywords}

CT, Third ventricle width (TVW), Third ventricle ratio (TVR) 


\section{INTRODUCTION}

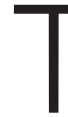
he human cerebral ventricular system contains a series of interconnecting CSF filled spaces and channels. Embryologically, ventricles originate from the central lumen of the neural tube and the cerebral vesicles. The advent of CT scanning resolved the problem of volumetric measurement performed on normal subjects. ${ }^{1}$

The ventricular system consists of two lateral ventricles, a third ventricle and a fourth ventricle. The two lateral ventricles communicate through the inter-ventricular Foramina of Monro with the third ventricle. Blockage of cerebral aqueduct can lead to widening of third ventricle and accumulation of excess amount of CSF in $3^{\text {rd }}$ and lateral ventricles leading to a condition known as hydrocephalus. Third ventricle is related to many important brain structures including anterior commissure, fornix, telachoroidea, pineal body, hypothalamic structures, optic chiasma, infundibulum, mammillary bodies, and tegmentum of midbrain. ${ }^{2}$ Therefore it can be easily obstructed by brain tumors in these regions and thus cause hydrocephalus and raised intracranial pressure. Buildup of pressure in third ventricle can also cause hypothalamic symptoms like diabetes insipidus and obesity. Besides, ventricular enlargement has been associated with many neuro-degenerative disorders e.g. brain atrophy diseases, basal ganglia atrophy, Huntington's chorea, alcoholic brain dementia etc. This enlargement may be primary or secondary to these pathological conditions.

The importance of base line data of ventricle measurements in normal living human for diagnosing and monitoring of several pathologies cannot be denied. Third ventricle width has been reported by various authors as $2.29 \mathrm{~mm}$ to 9.2 $\mathrm{mm}^{3-6}$ Measurement of ventricles of the brain in Multidetector Computed Tomography is important for evaluating changes due to growth, ageing, intrinsic and extrinsic pathologies. ${ }^{7}$ The ventricles of the brain are well visualized, and their overall configuration can be reconstructed from a series of contiguous slices. ${ }^{7}$ The evaluation of the normal measurements of the cerebral ventricles in the living human has great importance in the diagnosis and monitoring of several pathologies. ${ }^{8}$ Accurate measurements of the ventricles provide available and safe means of aiding the diagnosis of some neurological disorders such as early detection of hydrocephalus and cerebral atrophy and provide important follow up information in affected patients. ${ }^{9}$

The aim of present morphometric study is to examine the normal data range of Normal third ventricle width at a level of superior colliculus, third ventricle sylvian fissure index at a level of thalamus, third ventricle ratio at the level of Foramen of Monro, antero posterior and transverse diameter at a level of head of caudate nucleus.

The objectives were to measure the third ventricle width, third ventricle sylvian fissure distance index (TSFI) and third ventricle ratio (TVR), and to correlate third ventricle width with AP diameter and transverse diameter (TD) of cerebrum, and to determine correlation of third ventricle width with gender and age of patient.

\section{METHODS}

This study was conducted in the Department of Radiology and Imaging, Tribhuvan University Teaching Hospital, Kathmandu, Nepal during the period from July to October 2019.

The study design was quantitative, retrospective, cross sectional. CT scan was done by Somatom Definition $\mathrm{AS}+$. All the CT scan of head without pathological findings during the study period were enrolled numbering 100 total patients. Data was collected from the measurement performed on CT scan of head on axial sections.

Adult patients of both genders, $\geq 18$ years of age referred for CT scan of head without pathological findings were included in the study. Patients having pathological changes such as Alzheimer's disease, intraventricular hemorrhage, ventriculomegaly, meningitis, concussion and any other cerebral deformities which may interfere with normal morphology of the ventricle and patients with cerebral atrophy were excluded from the study.

The dimensions of third ventricle were measured in axial section of CT head.

In axial slice,

- The maximum width of third ventricle (TVW) was measured at a level of superior colliculus.

- The third ventricle sylvian fissure index (TSFI) was measured at a level of Thalamus. (TSFI $=r+$ I/D)

- The third ventricle ratio (TVR) was measured at a level of foramen of monro. (TVR=ThF/DF)

- The anteroposterior (AP) and transverse diameter (TD) was measured at a level of head of caudate nucleus, because at this level cerebrum dimensions are maximum and image at this level is available in almost all the patient.

Statistical analysis was carried out with the help of IBM SPSS version 25 software. The mean, SD and comparison of different dimensions of third ventricle were expressed in tables and bar diagrams. Data were presented as mean and SD for all variables. Patient's age, gender, TVW, TSFI, TVR, AP, TD were recorded. Data were obtained and analyzed using the descriptive statistics to summarize the information, and inferential statistics (paired sample t-test) and spearman's correlation coefficient to compare mean if there were significant difference in patient's gender (male and female). P value less than 0.05 was considered as level of significance. 


\section{RESULTS}

The data was collected from 100 subjects found to be normal in 50 males and 50 females with the age from 18 to 90 years old. Out of 100 patients, 50 were male and 50 were female.

Among the images of 100 normal subjects, the minimum age was 18 years and the maximum age was 87 years. The mean age of the patients was 45.53 years. Out of the total patients, 16 were in age group 18-27, 16 were between age group 28-37, 25 were between age group 38-47, 18 were between age group 48-57, 17 were between age group 5867, 6 were between age group 68-77, and 2 were between age group 78-87. The maximum number of patients were found in the age group78-87.

The mean $\pm S D$ of third ventricle width was $0.35 \pm 0.09 \mathrm{~cm}$, third ventricle sylvian distance index mean $\pm S D$ was $0.41 \pm 0.67 \mathrm{~cm}$ and third ventricle ratio mean $\pm S D$ was $0.03 \pm 0.01 \mathrm{~cm}$.

The third ventricle width showed very weak negative non-significant correlation with anteroposterior and transverse diameter.The ventricle sylvian fissure distance index and Third ventricle ratio showed non-significant correlation with anteroposterior and transverse diameter.

The mean \pm SD age of patient was $45.53 \pm 15.24$ year. The spearman's correlation coefficient between TVW and age was 0.32 and the distribution had moderate positive correlation with age.

The mean $\pm S D$ of third ventricle width was $0.33 \pm 0.09 \mathrm{~cm}$. The spearman's correlation between TVW and AP showed very weak negative nonsignificant correlation. The spearman's correlation between TVW and TD showed very weak nonsignificant correlation.

\section{DISCUSSION}

The aim of this study was to estimate the normal data range of third ventricle width, third ventricle sylvian fissure index and third ventricle ratio and to assess its association with gender and age. Narrowing of cerebral aqueduct can lead to third ventricle enlargement. Third ventricle can also be dilated in atrophic diseases of grey matter including basal ganglion e.g. multiple sclerosis. Therefore, the relation of the cerebral ventricular system to that of the brain tissue as a whole may provide much reliable information regarding cerebral ventricular diseases.
Table 2. Different ventricle parameters correlation with $A P \& T D$

\begin{tabular}{lcccc}
\hline & \multicolumn{2}{c}{ With AP diameter } & \multicolumn{2}{c}{$\begin{array}{c}\text { With transverse } \\
\text { diameter }\end{array}$} \\
\hline Parameter & $\mathbf{r}$ & $\mathbf{p}$ & $\mathbf{r}$ & $\mathbf{p}$ \\
\hline TVW & -0.009 & 0.93 & 0.12 & 0.23 \\
TSFI & 0.17 & 0.80 & 0.05 & 0.96 \\
TVR & 0.25 & $0.05^{*}$ & -0.17 & 0.80 \\
\hline
\end{tabular}

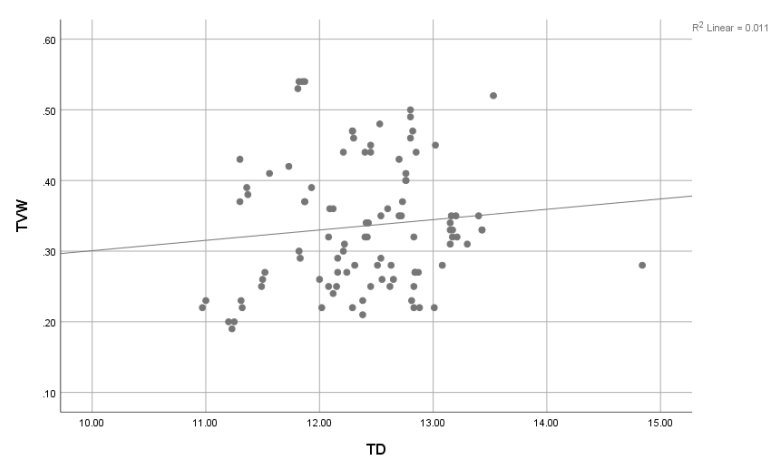

Fig 1. Scatter plot of width of third ventricle in $\mathrm{cm}$ \& anteroposterior diameter

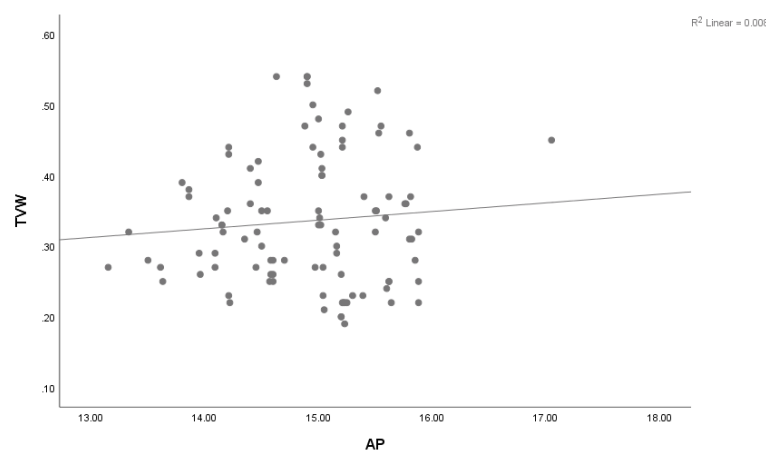

Fig 2. scatter plot of width of third ventricle in $\mathrm{cm}$ \& transverse diameter

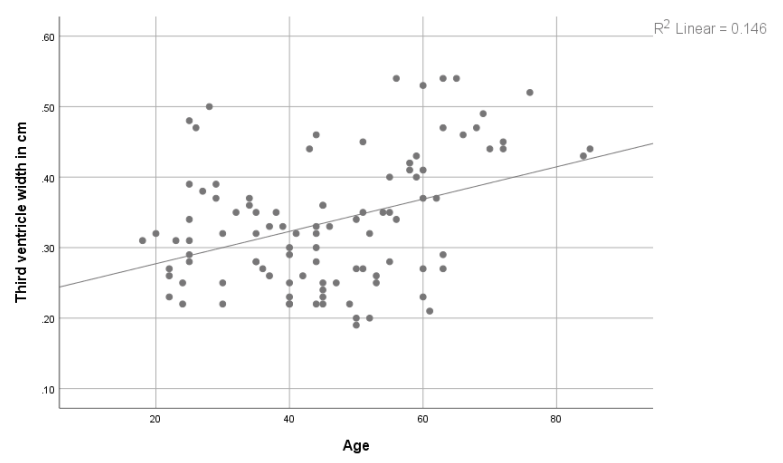

Fig 3. scatter plot of width of third ventricle in $\mathrm{cm} \&$ age in year

Table 1. Distribution of Mean value, S.D and P value in male and female.

\begin{tabular}{lccc}
\hline \multicolumn{1}{c}{ Parameter } & Overall & Male & Female \\
\hline Maximum diameter of third ventricle (TVW) & $0.35 \pm 0.09$ & $0.35 \pm 0.10$ & $0.31 \pm 0.06$ \\
Third ventricle sylvian fissure distance index (TSFI) & $0.41 \pm 0.67$ & $0.42 \pm 0.06$ & $0.39 \pm 0.06$ \\
Third ventricle Ratio (TVR) & $0.03 \pm 0.01$ & $0.03 \pm 0.01$ & $0.02 \pm 0.01$ \\
\hline
\end{tabular}


The sample size of the study consisted of 100 patients undergoing $\mathrm{CT}$ scan of head age ranged from 18 to 87 years. Among 100 samples, 50 were male and 50 were female consisting of $50 \%$ each respectively. Descriptive statistics of different measured values were presented in Table 1, 2, and 3. The mean age of patients was 45.53 years with standard deviation of $\pm 15.24 \mathrm{~cm}$.

In our study the mean of third ventricle width (TVW) was $0.3 \pm 0.09 \mathrm{~cm}$ at a level of superior colliculus. The mean of third ventricular width was $0.35 \pm 0.10 \mathrm{~cm}$ in male and $0.31 \pm 0.06 \mathrm{~cm}$ in female respectively. The values were slightly higher in males. A similar type of study performed by Gameraddin et al. and found the width of the third ventricle was $5.70 \pm 1.54$ $\mathrm{mm}$ and $5.40 \pm 1.68 \mathrm{~mm}$ in the males and females respectively.10 Similarly another study conducted by Patnaik et al observed the mean of third ventricle width was $6.86 \pm 2.74 \mathrm{~mm} .{ }^{11} \mathrm{~A}$ another study performed by D'Souza and Natekar observed the width of the third ventricle was $0.45 \pm 0.29$ $\mathrm{cm}$ and $0.39 \pm 0.17 \mathrm{~cm}$ in males and females respectively. ${ }^{12}$ Earlier also TVW was reported to be slightly higher in males than in females. ${ }^{12,13,14}$ in all Indian studies. However Vidya K in MRI study reported TVW to be same in males and females. ${ }^{6}$ In our study when correlated to linear diameter of cerebrum, TVW showed vey weak negative non-significant correlation to anteroposterior and transverse diameter. The spearman's correlation coefficient between age and third ventricle width was 0.32 . The third ventricular width had moderate positive correlation with age and also statistically significance with age (P value 0.01$)$ and gender $(P$ value 0.01 ) in male. In our study the width of third ventricle was found slightly lower and the difference might be due to the ethnicity and demography of the population.

The mean of third ventricle sylvian fissure distance index was $0.41 \pm 0.67 \mathrm{~cm}$ at a level of thalamus. The mean of third ventricle sylvian fissure distance index was $0.42 \pm 0.65 \mathrm{~cm}$ in male and $0.39 \pm 0.06$ $\mathrm{cm}$ in female. The values were slightly higher in male. TSFI showed significant correlation with transverse diameter and showed weak nonsignificant with anteroposterior diameter of cerebrum. TheThird ventricle sylvian fissure ratio index serves as an index on brain CT to give the predictive value for improvement after shunt surgery in elderly patients with normal pressure hydrocephalus based upon their symptoms. Large sizes of the ventricles, periventricular lucency and the presence of a small degree or the absence of cortical atrophy are considered characteristic evidence of communicating hydrocephalus. ${ }^{13,14}$ Thus there is an effect of the size of the ventricles and the cerebral mantle measured in CT images, in the presentations of clinical symptoms and the outcome of patients after drainage procedures.
The depth of the cerebral mantle, calculated by TSFI has a significant correlation to the severity of the symptom 'gait disturbances'. This is again probably the result of dilatation of third ventricle, which causes displacement and stretching of the fibres of the internal capsule, which originate from the precentral motor cortex. Additionally, alterations in the vascularity of the periventricular parenchyma structure can explain the mobility dysfunction. ${ }^{13}$

In our study, the mean of third ventricle ratio was $0.03 \pm 0.09 \mathrm{~cm}, 0.03 \pm 0.09 \mathrm{~cm}$ in male and $0.02 \pm 0.09$ $\mathrm{cm}$ in female respectively at a level of foramen of Monro.The value were slightly higher in male than in female.TVR showed statistically significant with anteroposterior diameter and weak negative nonsignificant with transverse diameter. Third ventricle measurement at this level is supposed to be a good indication of third ventricle enlargement. Further, tumor or cyst at this region can lead to dilation of Lateral ventricles causing pressure changes also in cerebrum. All the three parameters of third ventricle do not depend upon the anteroposterior diameter of cerebrum. Anterior inferior $3^{\text {rd }}$ ventricle is dilated in advanced hydrocephalus, but it remains normal in atrophy patients. The appearance of the anterior third ventricle is a helpful feature in distinguishing atrophy from CSF obstruction. ${ }^{16}$ The posterior third ventricle and upper aqueduct may also dilate with significant atrophy of the quadrigeminal plate. ${ }^{17}$ Therefore it is essential that measurements of third ventricle are available at all the level superior colliculus, thalamus and foramen of monro, so that appropriate diagnosis and management can be initiated

\section{CONCLUSION}

The third ventricle width in $\mathrm{CT}$ in this pilot study was $0.33 \pm 0.09 \mathrm{~cm}$. CT was very useful to determine the upper limits of normal value and its variation with age.

The study should be performed in larger sample size and age groups to make nomogram of Nepalese population. Further study on third ventricle in sagittal plane should be performed in relation to anteroinferior part, posterior part and mamillopontine distance, so that comprehensive data should available which will be rewarding for surgical procedure like third ventriculostomy and intraventricular placement of implants in future

\section{CONFLICT OF INTEREST}

None declared.

\section{REFERENCES}

1. Hounsfield GN. Computerized transverse axial scanning (tomography): part 1. Description of system, BJR, 1973;46: 101622. 


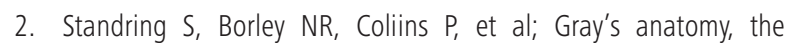
anatomical basis of clinical practice, 40th edition, Spain, Elsevier, 2008; 237-240.

3. Gawler J, Du Boulay GH, Bull JWD, et al. Computerized tomography (the EMI Scanner): a comparison with pneumoencephalography and Ventriculography, J. Neurol. Neurosurgy. Psychiatry, 1976; Volume 39, 203-211.

4. Soininen $H$, Puramen $M$, and Riekkinen PJ, Computed tomography findings in senile dementia and normal ageing, J. Neurol. Neurosurgy, Psychiatry, 1982; Volume 45, 50-54.

5. Zauhair A. Jaumah Fadhil A. AL-Kafhaji, C.T Scan Measurements of the Lateral and Third Ventricles in Apparently Normal Iraqi Subjects J Fac Med Baghdad Vol. 2009; 51, №.3.

6. Vidya K Satapara et al. Morphometry of width of Third ventricle of brain by luminal cast plastination \& MRI. IJAR. 2014; Vol 2 (6):837842.

7. Penn RD, Belanger WG, Yasnoff WA. Ventricular volume in man computed from CAT scans. Ann. Neurology, 1978:3:316-323.

8. Sabattini L, Evaluation and measurement of the normal ventricular and subarachnoid spaces by CT. Neuroradiology; 1982; 23:1-5.

9. Delsi L. E, Perman G. P, Targus S. D et al. CT in chronic schizophrenia form disorder and other acute psychiatric disorders, Arch. Psych; 1982;39:778-783.
10. Black PM, Ojemann RG, Tzouras A. CSF shunts for dementia, incontinence, and gait disturbance. Clin Neurosurg. 1985;32:632651.

11. Borgesen SE, Gerris F. The predictive value of conductance to outflow of cerebrospinal fluid in normal pressure hydrocephalus. Brain. 1982; vol. 105: pg 65- 86

12. Weller RO, Shulmann K. Infantile hydrocephalous: Clinical, Histological and ultrastructurally study of brain damage. J Neurosurgery, 1972, vol 36: 255-265.

13. Gammal TE, Allen MB Jr, Brooks BS, et al. MR Evaluation of hydrocephalus. AJR Am J Roentgenol. 1987 Oct;149(4):807-13.

14. El Gammal T. Extraventricular (communicating) hydrocephalus: Some observations on midline ventricles AJR. 1969, vol 106, 308328.

15. Keith L. Moore, Clinically Oriented Anatomy, 7th Edition. New York: Lippincott Williams \& Wilkins: 2014. p. 879-886.

16. Mathew LS, Paul M, Bakshi S. Assessment of third ventricle with MRI: morphological variations among different gender and age group. Webmed Central Anatomy 2012;3(5):WMC003370

17. Usman JD, Zagga AD, Tadros AA, et al. Morphological variation of third ventricle using computerized tomography among different gender and age groups: A 5-year retrospective study in Usmanu Danfodiyo University Teaching Hospital, Sokoto, North - West Nigeria. Sahel Med J 2013;16:83-6. 